\title{
Introduction to the Wireless Networks minitrack
}

\author{
Edoardo Biagioni \\ University of Hawaii at Manoa \\ esb@hawaii.edu
}

\author{
John McEachen \\ Naval Postgraduate School \\ mceachen@nps.edu
}

\author{
Murali Tummala \\ Naval Postgraduate School \\ mtummala@nps.edu
}

\section{Wireless Networks}

Even just a few years ago, most of us checked our email and browsed the web on a desktop or laptop computer. These days, most people live an alwaysconnected life that relies heavily on wireless networks.

Unfortunately and unlike wired networks, wireless networks are not always available, offer variable and often unpredictable quality of service, and sometimes come at a substantial cost. Even the "alwaysconnected" nature of wireless networks sometimes affects people's enjoyment of their lives. In short, wireless networks are a fertile area for research.

\section{Papers in this minitrack}

This minitrack has five papers, beginning with a paper that presents a design to reduce handover delay by storing the location of mobile hosts in a hierarchical Distributed Hash Table. This new design has been evaluated on Mininet and a commercial cloud to demonstrate handovers of less than $120 \mathrm{~ms}$.

The next two papers study network latency in IP and GPRS networks. The first of these determines the latency within a large automated (machine-to-machine) GPRS-based network by studying logs of TCP connections which record the time at which data is acked, and the time at which the connection is closed. The median of this observed time difference is about $1550 \mathrm{~ms}$ to close the connection, validating earlier studies that report 900ms median Round-Trip-Time, on a scale including almost 30 times more systems than in earlier studies. The paper presents further observations of the behavior of TCP, including the retransmission timer and the binary exponential backoff.

The third paper is related to the second, but focuses on modeling the network latency using different statistical distributions, finding that "the double Paretolognormal distribution and the generalized Beta distribution of the second kind fit the data equally well."

The fourth paper focuses on the difficulty of maintaining privacy in urban cellular networks, and examines the effects of deriving network information on specific users based on timing advance and temporary identifiers.

The fifth paper analyzes the business potential of deploying $5 \mathrm{G}$ networks in urban, suburban, and rural areas, specifically considering the population distribution in the state of Texas. Using the Diffusion of Innovation model parametrized by historical data of broadband adoption, this paper projects that deployment of $5 \mathrm{G}$ can be profitable to operators, and further analyzes the sensitivity of this result to each of the assumptions required for the estimate.

\section{About this minitrack}

This minitrack was first held in 2005, and the track chairs are unchanged since 2011. As can be seen by the selection of this year's papers, we welcome submissions on any aspect of wireless networks technical, of course, but also social, economic, cultural, and others. 